\title{
NOVOS HÍBRIDOS BASEADOS EM OXIHIDRÓXIDO DE FERRO(III) E NANOPARTÍCULAS DE OURO (AuNPs/ FeOOH) COMO CATALISADORES PARA A REDUÇÃO DE POLUENTES ORGÂNICOS AMBIENTAIS
}

\author{
Camila Kauany da Silva Azevedo ${ }^{a}$, Eduardo Arizono dos Reis ${ }^{\mathrm{a}}$, José Carlos Germino ${ }^{\mathrm{b}}$, Jéferson Aparecido Moreto , Ailton $^{\mathrm{c}}$ \\ José Terezo $^{\text {a e Fernando Júnior Quites }}{ }^{\text {a,*,\# }}$ \\ aDepartamento de Química, Instituto de Ciências Exatas e da Terra, Universidade Federal de Mato Grosso, $78060-900$ Cuiabá - \\ MT, Brasil \\ 'Instituto de Química, Universidade Estadual de Campinas, 13083-970 Campinas - SP, Brasil \\ 'Departamento de Física, Instituto de Ciências Exatas, Naturais e Educação, Universidade Federal do Triângulo Mineiro, Avenida \\ Doutor Randolfo Borges Júnior - Univerdecidade, 75901970 Uberaba - MG, Brasil
}

Recebido em 24/10/2016; aceito em 22/02/2017; publicado na web em 24/04/2017

\begin{abstract}
NEW HYBRIDS BASED ON IRON (III) OXYHYDROXIDE AND GOLD NANOPARTICLES (AuNPs / FeOOH) as CATALYSTS FOR THE REDUCTION OF ORGANIC ENVIRONMENTAL POLLUTANTS. A new $\delta$-FeOOH supported nano-Au catalyst was prepared by a facile method using $\mathrm{HAuCl}_{4}$ as the gold source and $\mathrm{NaBH}_{4}$ as the reducing agent. The samples (denoted as AuNPs/FeOOH) were characterized by means of X-ray diffraction (XRD), UV-vis and infrared spectroscopies and scanning electron microscope (SEM). The obtained characterization data confirmed the formation of Au nanoparticles on the $\delta$-FeOOH matrix surface. SEM observations showed that Au nanoparticles have a spherical morphology and good dispersion with a narrow size distribution on the surface of iron (III) oxyhydroxide. The catalyst exhibits significantly high catalytic activity for hydrogenation of 4-nitrophenol (4-NP) to 4-aminophenol (4-AP) at room temperature. The high performance obtained can be attributed to the specific characteristics of the nanostructure of the catalyst of nano-Au deposited on the external surface of $\delta$ - $\mathrm{FeOOH}$, including plenty of oxygen-containing groups of $\delta$ - $\mathrm{FeOOH}$ for anchoring AuNPs. Moreover, the catalytic activity increased when the AuNPs content in the $\delta$-FeOOH support. The AuNPs-based $\delta$-FeOOH hybrids are interesting catalysts for hydrogenation of nitrophenols to aminophenols.
\end{abstract}

Keywords: gold nanoparticles; iron (III) oxyhydroxide; catalysis; nitrophenols.

\section{INTRODUÇÃO}

Compostos nitrofenólicos estão entre os poluentes mais encontrados em efluentes industriais e agrícolas. ${ }^{1}$ Estes compostos possuem elevada toxicidade, mesmo em níveis residuais, além de elevada solubilidade e estabilidade em meio aquoso. ${ }^{2}$ Entre os compostos nitrofenólicos, o 4-nitrofenol (4-NP) é considerado um poluente prioritário devido ao seu potencial genotóxico e carcinogênico aos seres humanos tornando importante sua conversão a compostos que possuem menor toxicidade. ${ }^{3}$ A redução do $4-\mathrm{NP}$ gera como produto o 4-aminofenol (4-AP), que comparado ao primeiro possui menor potencial tóxico e maior biodegradabilidade, além de ser um importante intermediário na indústria química. ${ }^{4,5}$

Materiais híbridos baseados em suportes inorgânicos e nanopartículas metálicas ${ }^{2,3,5}$ (NPs) são compostos que possuem diversas aplicações, entre elas, a utilização como catalisadores para a degradação e ou redução de poluentes orgânicos ambientais como o 4-NP. Esses materiais podem apresentar combinações de propriedades únicas que não são encontradas em compósitos convencionais. ${ }^{5,6}$ Além de obter resultados catalíticos melhorados, é possível também conseguir novas funcionalidades devido aos efeitos sinérgicos entre os seus diferentes componentes. ${ }^{7}$

Neste sentido, semicondutores oxihidróxidos de ferro (III) (FeOOH) tem atraído interesse na sua utilização como precursor para a produção de óxidos de ferro e como suportes para aplicações em catálise ${ }^{8}$ Os polimorfos do $\mathrm{FeOOH}$ são geralmente cristalinos e ocorrem em diferentes estruturas cristalográficas incluindo a goethita $(\alpha-\mathrm{FeOOH})$, akaganeíta $(\beta-\mathrm{FeOOH})$, lepidocrocita $(\gamma-\mathrm{FeOOH})$ e a

*e-mail: fquitesquim@gmail.com

\#e-mail alternativo: fquites@ufmt.br ferroxiíta $(\delta-\mathrm{FeOOH})$, que podem ser facilmente caracterizadas através da técnica de difração de raios $\mathrm{X}^{8}{ }^{89} \mathrm{O}$ método de preparação dos oxihidróxidos de ferro (III) é muito sensível às condições da síntese, na qual o tipo e a concentração do sal de ferro, o pH, a temperatura e o tempo de síntese (envelhecimento) são fatores importantes. ${ }^{8}$

Entre as fases de oxihidróxido de ferro (III), a fase ferroxiíta $(\delta-\mathrm{FeOOH})$ tem atraído especial atenção devido a sua estabilidade química, comportamento ferromagnético e propriedade bioquímica associada aos íons Fe(III) e seus orbitais 3d. , $^{7-11}$ Este composto é um polimorfo metaestável do oxihidróxido de ferro (III) com uma estrutura baseada em um empacotamento hexagonal dos átomos de oxigênio com os íons $\mathrm{Fe}$ (III) ocupando metade dos interstícios octaédricos disponíveis, similar à rede da hematita $\left(\mathrm{Fe}_{2} \mathrm{O}_{3}\right)$. A presença destes sítios octaédricos desigualmente ocupados induz uma magnetização espontânea na estrutura do $\delta$ - $\mathrm{FeOOH}$, com uma temperatura de Curie próxima a temperatura ambiente, possibilitando que este composto também seja empregado na medicina. ${ }^{7,9}$ Além disso, a banda de absorção localizado na região próxima a $600 \mathrm{~nm}$ induz um comportamento semicondutor a este material, com energia de band gap em torno de $2,2 \mathrm{eV}$, o que possibilita a utilização da luz solar como fonte de excitação para aplicações em fotocatálise heterogênea empregando luz na região do visível. ${ }^{12,13} \mathrm{~A}$ síntese deste material nanoparticulado é fácil e de baixo custo, além disso, as nanopartículas podem ser facilmente dispersas em água, o que aumenta a sua eficiência catalítica. ${ }^{10,12} \mathrm{O}$ $\delta$-FeOOH tem sido utilizado também como fotocatalisador para a degradação de poluentes orgânicos ambientais. ${ }^{9,10,12,14}$ Entretanto, seu emprego é ainda limitado devido às elevadas taxas de recombinação do par elétron-buraco. Uma alternativa para minimizar este efeito é a formação de híbridos com nanopartículas de metais nobres como $\mathrm{Ag}$, Au e Pt. Além de auxiliar a separação de cargas dos pares elétron-buraco, as nanopartículas metálicas também podem aumentar a 
atividade catalítica devido aos efeitos da banda de ressonância plasmônica de superfície. ${ }^{15} \mathrm{~A}$ utilização do suporte inorgânico também evita a aglomeração das nanopartículas metálicas, já que no estado sólido as mesmas tendem a se aglomerar reduzindo a sua atividade catalítica. ${ }^{3,16}$ Entre as nanopartículas metálicas, as nanopartículas de ouro (AuNPs) têm atraído muito interesse devido às suas propriedades ópticas e eletrônicas que favorecem seu emprego em diversas áreas como na catálise, fabricação de sensores e aplicações biomédicas. ${ }^{10}$ Neste sentido, Yue e colaboradore ${ }^{17}$ demostraram a síntese de nanocompósitos baseados em $\beta$-FeOOH (fase akaganeíta) e nanopartículas de ouro (AuNPs) para a detecção de traços de melanina em solução aquosa. Neste estudo foi observado que as nanopartículas de ouro foram adsorvidas na superfície do suporte $\beta-\mathrm{FeOOH}$ devido às intensas interações entre a hidroxilas superficiais do hospedeiro inorgânico com a superfície das AuNPs.

Uma aplicação interessante para estudar a estabilidade e atividade catalítica de nanopartículas metálicas suportadas em óxidos inorgânicos é a redução catalítica de 4-nitrofenol a 4-aminofenol usando borohidreto de sódio $\left(\mathrm{NaBH}_{4}\right)$ como agente redutor em solução aquosa. ${ }^{5,18,19}$ Esta reação é facilmente monitorada pela espectroscopia óptica de absorção molecular na região do UV-vis. Embora este modelo de reação catalisada por nanopartículas metálicas seja muito bem entendido, ele representa uma necessidade real no mundo, uma vez que 4-NP é um poluente comum em efluentes aquosos, e seu produto reduzido, o 4-aminofenol (4-AP), é um precursor orgânico muito usado em diferentes reações químicas. Esta reação demonstra a transformação de nitroaromáticos em anilinas funcionalizadas, as quais apresentam significante utilidade industrial. NPs de Au, Ag, Pt e Pd são ativas cataliticamente para esta reação de redução de compostos nitrogenados orgânicos. ${ }^{5,14,19}$ Recentemente, Damasceno e colaboradores ${ }^{20}$ ocluíram nanopartículas de ouro e de paládio e suas nanoligas (AuPd) nos canais mesoporosos da sílica SBA-15. Estes materiais híbridos foram empregados como catalisadores para a redução de 4-NP a 4-AP na presença de $\mathrm{NaBH}_{4}$. De acordo com os estudos observados por estes autores, foi vista uma correlação entre a atividade catalítica e a quantidade de paládio inserida nas nanoligas de AuPd depositadas no suporte SBA-15, ou seja, a medida que o teor de NPs de paládio aumentou nas ligas (AuPd), a atividade catalítica dos híbridos AuPd/SBA-15 também foi aumentada. Estes resultados indicaram que a redução de 4-NP pode ser facilmente controlada através dos constituintes presentes na liga AuPd nos catalisadores AuPd/SBA-15. Ainda em relação às nanopartículas metálicas, elas também têm sido depositadas na superfície de materiais magnéticos (como por exemplo, $\mathrm{Fe}_{3} \mathrm{O}_{4}$ ou $\mathrm{Fe}_{2} \mathrm{O}_{3}$ ) para aplicações catalíticas em diversas reações orgânicas, como pode ser visto no excelente artigo de revisão publicado recentemente por Rossi e colaboradores. ${ }^{21}$ Uma estratégia muito empregada na literatura têm sido a preparação de caroços magnéticos revestidos superficialmente com sílica $\left(\mathrm{SiO}_{2}\right){ }^{21,22}$ Como a superfície da sílica é facilmente funcionalizada, grupos funcionais podem ser ligados covalentemente em sua estrutura permitindo, em uma etapa posterior, a deposição de íons metálicos seguida por sua redução para a formação das NPs metálicas. ${ }^{21,22}$ Woo e Park $^{23}$ depositaram AuNPs na superfície de microesferas de magnetita $\left(\mathrm{Fe}_{3} \mathrm{O}_{4}\right)$ revestidas com sílica e polímeros orgânicos. A atividade catalítica das AuNPs nestes híbridos $\left(\mathrm{Fe}_{3} \mathrm{O}_{4} @ \mathrm{SiO}_{2} @\right.$ Polímero@AuNPs $)$ foi empregada para a reação de hidrogenação do 4-NP a 4-AP, na qual os catalisadores foram facilmente separados do meio reacional usando separação magnética. $\mathrm{Fe}_{3} \mathrm{O}_{4}$ revestida com $\mathrm{SiO}_{2}\left(\mathrm{Fe}_{3} \mathrm{O}_{4} @ \mathrm{SiO}_{2}\right)$ foi também usada para a preparação de compostos híbridos contendo quitosona e nanopartículas de prata (AgNPs), como demostrado por Wang e colaboradores. ${ }^{24}$ As AgNPs mostraram atividade catalítica para a hidrogenação do 4-NP a 4-AP na presença de $\mathrm{NaBH}_{4}$, na qual, de acordo com estes autores, a redução total do 4-NP aconteceu em aproximadamente 3 min de reação. Walker e Zaleski ${ }^{25}$ também demostraram a preparação de híbridos multifuncionais feitos de $\mathrm{Fe}_{3} \mathrm{O}_{4} @ \mathrm{SiO}_{2}-\mathrm{M}$, em que $\mathrm{M}$ se refere às nanopartículas de ouro, prata e paládio para a redução catalítica de 4-nitrofenol.

Baseando-se nestes estudos, pode-se concluir que o grande interesse nas fases de oxihidróxido de ferro (III) é que elas podem ser usadas como precursoras para a obtenção de óxidos de ferro quimicamente e termodinamicamente mais estáveis a temperaturas ambientes como, por exemplo, a hematita $\left(\mathrm{Fe}_{2} \mathrm{O}_{3}\right){ }^{8,17,26}$ Outra vantagem associada ao uso destes materiais, em relação às fases dos óxidos de ferro, é que defeitos superficiais ou poros estruturais podem facilmente existir ou serem criados com maior facilidade na superfície do $\mathrm{FeOOH}$, tornando-a mais reativa e atrativa para o confinamento de átomos, moléculas ou nanopartículas em sua superfície. ${ }^{17,26}$ Sendo assim, a facilidade da preparação sintética de híbridos baseados em NPs metálicas/FeOOH faz com que eles se tornem atraentes precursores para a preparação de óxidos de ferro (III) decorados com nanopartículas metálicas através de um simples tratamento térmico. ${ }^{17,29}$

Neste sentido, com a intenção em preparar catalisadores híbridos, é demonstrado neste estudo uma rotina simples e fácil para a preparação de oxihidróxido de ferro (III) $(\delta$-FeOOH) decorado com nanoparticulas de ouro metálico (AuNPs) via um método de redução in situ em condições brandas de síntese. Esta estratégia pode também ser aplicada a outras NPs como Ag e Pt, ou partículas bimetálicas (como exemplo, AgAu e AuPt). A intenção em depositar as AuNPs na superfície do $\delta$-FeOOH é devido à banda ressonante plasmônica que as mesmas apresentam na região do visível e às suas propriedades catalíticas. ${ }^{16,27}$ Portanto, inicialmente os materiais híbridos AuNPs/FeOOH foram preparados e caracterizados estruturalmente e espectroscopicamente, e medidas de difração de raios X (DRX) e imagens de microscopia eletrônica de varredura (MEV), bem como medidas de absorção na região do UV-vis e infravermelho demonstraram a formação das NPs de ouro e sua deposição na superfície do FeOOH. Em seguida, a atividade catalítica das NPs de Au nos híbridos (AuNPs/FeOOH) foi também avaliada usando a redução do 4-NP a 4-AP na presença de $\mathrm{NaBH}_{4}$ à temperatura ambiente.

\section{PARTE EXPERIMENTAL}

\section{Materiais}

Os materiais utilizados neste trabalho foram: sulfato ferroso amoniacal $\left(\mathrm{Fe}\left(\mathrm{SO}_{4}\right)\left(\mathrm{NH}_{4}\right) \cdot 6 \mathrm{H}_{2} \mathrm{O}\right)$, hidróxido de sódio $(\mathrm{NaOH})$, peróxido de hidrogênio $\left(\mathrm{H}_{2} \mathrm{O}_{2}\right)$, ácido tetracloroaurato (III) $\left(\mathrm{HAuCl}_{4}\right)$, borohidreto de sódio $\left(\mathrm{NaBH}_{4}\right)$, 4-nitrofenol e água destilada.

\section{Síntese das nanopartículas de $\delta$-FeOOH}

Inicialmente, foi preparada uma solução de sulfato ferroso amoniacal $\left(\mathrm{Fe}\left(\mathrm{SO}_{4}\right)\left(\mathrm{NH}_{4}\right) \cdot 6 \mathrm{H}_{2} \mathrm{O}\right)\left(0,028 \mathrm{~g} \mathrm{~mL}^{-1}\right)$. Em um banho de ultrassom, gotejaram-se nesta solução $100 \mathrm{~mL}$ de uma solução de hidróxido de sódio $\left(2 \mathrm{~mol} \mathrm{~L}^{-1}\right)$, produzindo um precipitado verde. ${ }^{9,12}$ Em seguida, foi adicionado rapidamente $2,5 \mathrm{~mL}$ de peróxido de hidrogênio $\left(\mathrm{H}_{2} \mathrm{O}_{2}\right) 30 \%$ sob agitação constante. Imediatamente foi produzido um precipitado marrom avermelhado característico do $\delta$-FeOOH, que foi separado por centrifugação, lavado com água destilada e álcool etílico e seco em temperatura ambiente em dessecador. $\mathrm{O}$ material obtido nesta etapa foi nomeado como $\mathrm{FeOOH}$.

\section{Síntese dos nanohíbridos de AuNPs/FeOOH}

Os híbridos AuNPs/FeOOH foram sintetizados empregando 
diferentes quantidades de ouro. Em uma síntese típica: $300 \mathrm{mg}$ de FeOOH foram dispersos em $20 \mathrm{~mL}$ de uma solução etanólica de $\mathrm{HAuCl}_{4}$. A suspensão foi mantida em agitação por cerca de $1 \mathrm{~h}$. Em seguida, foram adicionados $5,0 \mathrm{~mL}$ de uma solução de $\mathrm{NaBH}_{4}$ $\left(0,01 \mathrm{~mol} \mathrm{~L}^{-1}\right)$ como agente redutor para reduzir os íons $\mathrm{Au}^{3+}$ à AuNPs na superfície do $\delta$-FeOOH. Esta mistura ficou em agitação por cerca de $18 \mathrm{~h}$ em temperatura ambiente. Após este período, o sólido foi separado por centrifugação, lavado com água destilada e álcool etílico e seco à temperatura ambiente. Foram utilizadas diferentes concentrações da solução etanólica de $\mathrm{HAuCl}_{4}$ : $2,5 \times 10^{-3} \mathrm{~mol} \mathrm{~L}^{-3}$

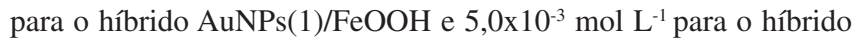
AuNPs(2)/FeOOH.

\section{Caracterização dos materiais}

A fim de se obter informações sobre a estrutura cristalina dos materiais, foram realizadas medidas de difração de raios X (DRX) via método do pó depositando as amostras sólidas em porta amostra de vidro. Os difratogramas foram obtidos em um difratômetro modelo D8 Advance Bruker com fonte de radiação de cobre $\mathrm{Cu}$ ( $\mathrm{K} \alpha=1,5418 \AA$ ) , com tensão de $40 \mathrm{kV}$ e corrente de $40 \mathrm{~mA}$. Os dados foram coletados na faixa de $20-70^{\circ} 2 \theta$ em intervalo de $0,01^{\circ}$ e velocidade de $4^{\circ} \mathrm{min}^{-1}$. Para analisar as propriedades ópticas dos materiais foram obtidos espectros de absorção molecular na região do ultravioleta e do visível (UV-vis) no modo de refletância difusa. Os espectros UV-vis foram registrados em um espectrômetro modelo Cary 50 acoplado com uma esfera de integração usando $\mathrm{BaSO}_{4}$ como referência. A morfologia dos nanocompósitos foi estudada através das técnicas de microscopia eletrônica de varredura. Os espectros de absorção na região do infravermelho com transformada de Fourier (FTIR) das amostras em $\mathrm{KBr}$ foram registrados na faixa de $4.000 \mathrm{a}$ $400 \mathrm{~cm}^{-1}$ em um espectrômetro Nicolet 6700. As imagens de MEV foram obtidas usando um microscópio eletrônico de varredura de alta resolução modelo JEOL-2010. As amostras foram dispersas em isopropanol, sonicadas por 30 min e depositadas em um porta-amostra metálico.

\section{Avaliação da atividade catalítica}

A atividade catalítica das nanopartículas de ouro nos híbridos (AuNPs/FeOOH) foi realizada através da reação de redução do 4-NP na presença de $\mathrm{NaBH}_{4}$ em temperatura ambiente. Para isto, um excesso de $\mathrm{NaBH}_{4}\left(0,5 \mathrm{~mL} ; 33,4 \mathrm{~g} \mathrm{~L}^{-1}\right)$ foi adicionado a $10 \mathrm{~mL}$ de uma solução aquosa de 4 -NP $0,21 \mathrm{~g} \mathrm{~L}^{-1}$ sob constante agitação. Após a mudança de coloração da solução, de amarelo claro a amarelo escuro, adicionaram-se $10 \mathrm{mg}$ do catalisador. A conversão de 4-NP a 4-AP foi monitorada espectrofotometricamente através de medidas de absorção molecular obtidas na faixa de 200-800 nm em um espectrofotômetro UV-vis modelo Varian Cary 50.

Modelos cinéticos para a redução do 4-nitrofenol na presença dos catalisadores foram empregados para descrever o progresso da reação catalítica. ${ }^{3,5}$

\section{RESULTADOS E DISCUSSÕES}

\section{Caracterização estrutural e espectroscópica dos híbridos $\mathrm{AuNP} / \mathrm{FeOOH}$}

As fases cristalinas do $\mathrm{FeOOH}$ e de seus híbridos (AuNPs/FeOOH) foram estudadas, primeiramente, pela técnica de difração de raios $\mathrm{X}$. Através da técnica de DRX, as fases cristalinas do oxihidróxido de ferro (III) podem ser facilmente identificadas. ${ }^{28}$ Os difratogramas do $\mathrm{FeOOH}$ e do material após a deposição das nanopartículas de ouro estão apresentados na Figura 1. Para o suporte FeOOH é observado picos de difração a $2 \theta$ em $35^{\circ} ; 40,5^{\circ} ; 54^{\circ}$ e $63^{\circ}$, os quais podem ser atribuídos aos planos de difração (100), (101), (102) e (110) característicos da fase ferroxiíta $(\delta-\mathrm{FeOOH})$ do oxihidróxido de ferro (III) (JCPDS N $\left.{ }^{\circ} 13-0087\right) .{ }^{9,12}$ Ainda, de acordo com o perfil de DRX, os parâmetros de rede para o $\delta$-FeOOH podem ser indexados a rede hexagonal (HC) com $a=b=2.95$ $\AA$ e $c=4.43 \AA$ (JCPDS N $\left.{ }^{\circ} 13-0087\right)$. ${ }^{7,9}$ Para o material híbrido AuNPs(2)/FeOOH podem ser vistos picos de difração a $2 \theta=36^{\circ}$; $40,5^{\circ} ; 54^{\circ}$ e $63^{\circ}$, correspondentes aos planos de difração da fase de $\delta$-FeOOH, ao passo que novos picos de difração aparecem a $2 \theta=38^{\circ}$, $44^{\circ}$ e $64^{\circ}$, que podem ser indexados às reflexões de Bragg (111), (200) e (220) (JCPDS Nº4-0784), respectivamente, relacionadas à fase cúbica de face centrada das nanopartículas de ouro depositadas na superfície do oxihidróxido de ferro (III). ${ }^{16}$ Usando a equação de Scherrer, o diâmetro médio estimado para as nanopartículas de ouro nesse material foi de $16 \mathrm{~nm}$, consistente com o diâmetro médio observado pelas imagens de microscopia (veja discussão mais adiante). Estes resultados também indicaram que as AuNPs foram depositadas na superfície do $\delta$-FeOOH e que as mesmas não perturbam a estrutura cristalina do suporte inorgânico, como já observado também no trabalho recentemente publicado por Yue e colaboradores. ${ }^{17}$

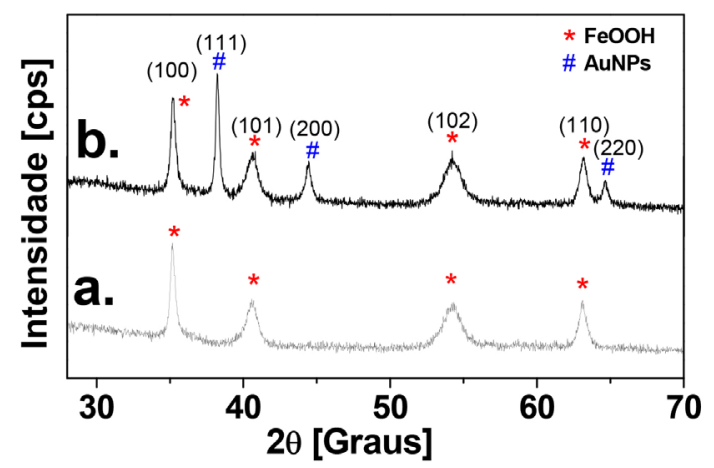

Figura 1. Difratogramas de raios $X($ a) do suporte $\delta$-FeOOH e de seu híbrido (b) AuNPs (2)/FeOOH. Os simbolos (*) e (\#) referem-se aos planos de difração das fases do $\delta$-FeOOH e do ouro metálico, respectivamente

As imagens de MEV mostraram que o suporte $\delta$-FeOOH foi sintetizado na forma de nanoplacas (Figura 2a), característico para materiais com a fase ferroxiíta $(\delta-\mathrm{FeOOH}){ }^{29,30}$ Para o composto híbrido $\operatorname{AuNPs}(2) / \mathrm{FeOOH}$ (Figura 2b-c), pode ser observada a presença de placas de $\delta$-FeOOH decoradas com "pontos" esféricos superficiais atribuídos às AuNPs depositadas em sua superfície. A morfologia esférica das nanopartículas de ouro adsorvidas na superfície do $\delta$-FeOOH foi também confirmada através da imagem MEV de alta resolução (veja Figura 2c). O espectro EDS-MEV e a distribuição atômica na superfície do híbrido AuNPs(2)/FeOOH mostrou que o mesmo possui em sua composição química ferro e ouro (veja Figura 1S no Material Suplementar).

As vibrações a curta distância dos materiais produzidos neste estudo foram investigadas usando a espectroscopia de absorção na região do infravermelho. A Figura 3 mostra os espectros FTIR do $\delta$-FeOOH e de seus híbridos. $\mathrm{O}$ espectro do $\delta$-FeOOH (Figura $3 a$ ) exibiu uma banda, com alta intensidade relativa e alargada, na região de $3260 \mathrm{~cm}^{-1}$, devido aos modos de estiramento de moléculas de água fissiosorvidas em sua superfície. A banda a $3130 \mathrm{~cm}^{-1}$ pode ser atribuída à hidroxila superficial do FeOOH.,31 Para os materiais híbridos (Figura 3b-c), a inserção das nanopartículas de ouro promoveu a supressão da banda em $3130 \mathrm{~cm}^{-1}$ e, ao mesmo tempo, o surgimento de uma banda em $2900 \mathrm{~cm}^{-1}$ com intensificação da banda em $3260 \mathrm{~cm}^{-1}$. Estas bandas são relacionadas ao estiramento $(-\mathrm{OH})$, indicando a presença de moléculas de água adsorvidas na superfície dos híbridos. ${ }^{8,31,32}$ De acordo 

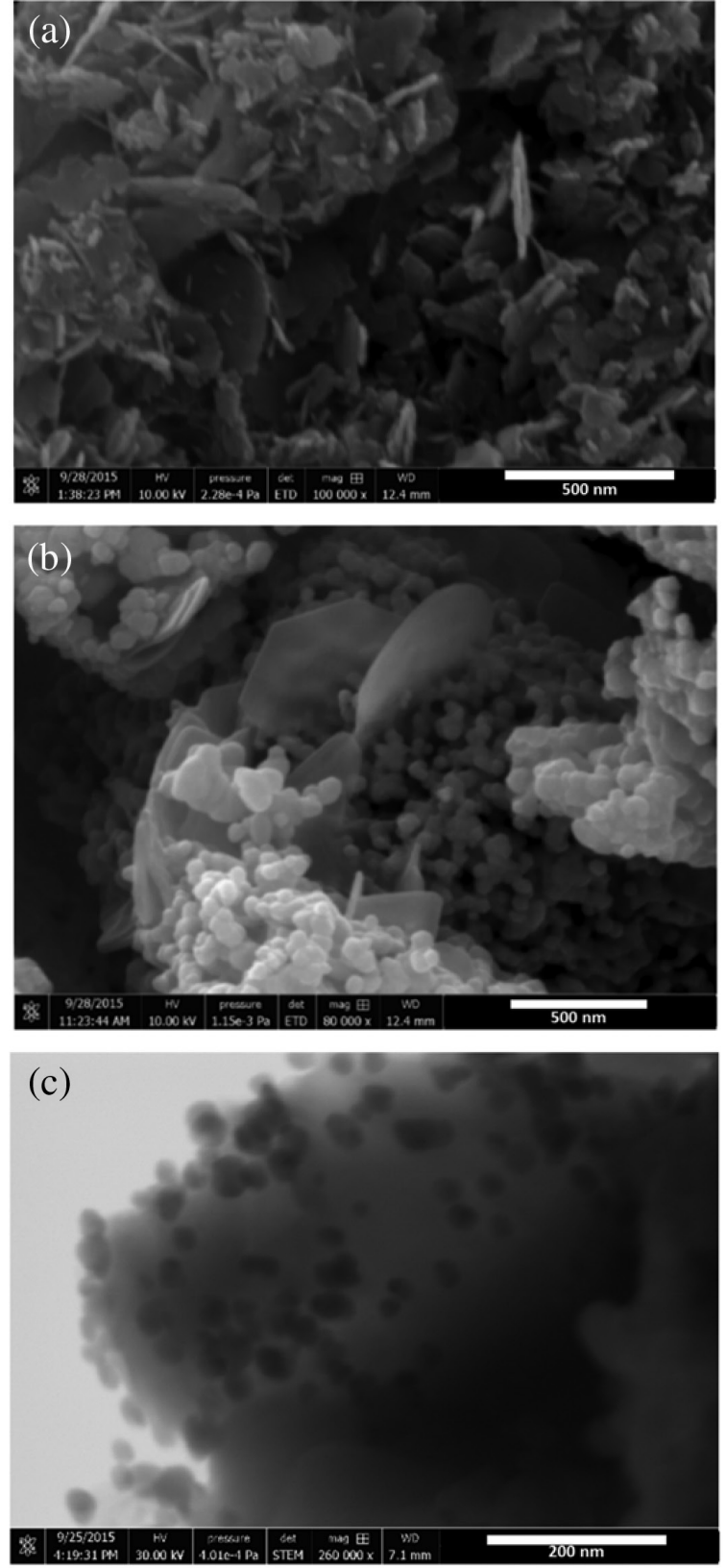

Figura 2. Imagens de microscopia eletrônica de varredura do suporte (a) $\delta$-FeOOH e do híbrido $\mathrm{Au}(2) / \mathrm{FeOOH}$ em (b) baixa resolução (barra de escala: $500 \mathrm{~nm}$ ) e (c) alta resolução (barra de escala: $200 \mathrm{~nm}$ )

com Villalba e colaboradores, ${ }^{32} \mathrm{o}$ alargamento desta banda pode ser também um indicativo de que não há diferenciação entre as vibrações das hidroxilas superficiais (-OH livres) e das hidroxilas no material como um todo (material "bulk") (-OH com ligações de hidrogênio intermolecular). Isto é uma possível evidência de que há uma interação entre as hidroxilas superficiais do $\delta$-FeOOH e as NPs de ouro nos materiais híbridos. Ainda em relação ao espectro do $\delta$-FeOOH puro, pode ser visto duas bandas a $1150 \mathrm{~cm}^{-1}$ e $890 \mathrm{~cm}^{-1}$ que correspondem às vibrações das ligações $\mathrm{Fe}-\mathrm{O}-\mathrm{OH} .{ }^{8,33}$ As bandas de transmitância na região de 500 a $780 \mathrm{~cm}^{-1}$ são características das ligações Fe-O. ${ }^{22,23}$ De acordo com a literatura, ${ }^{8,22,23}$ a banda próxima a $600 \mathrm{~cm}^{-1}$ é atribuída às vibrações de estiramento da ligação $\mathrm{Fe}-\mathrm{O}$ de íons $\mathrm{Fe}$ (III) ocupando sítios tetraédricos; e a banda a $440 \mathrm{~cm}^{-1}$ pode ser afixada às vibrações de estiramento de Fe-O de íons Fe(III) ocupando os sítios octaédricos da rede. As bandas em 780 e $892 \mathrm{~cm}^{-1}$ (Figura 3a) podem ser afixadas aos modos de vibração da $\delta_{(\mathrm{OH})}$ no plano e fora do plano do $\delta-\mathrm{FeOOH}$, respectivamente. Estas mesmas bandas discutidas acima estão deslocadas para mais alta energia nos materiais híbridos (Figura 3b-c). De acordo com Pedrosa e colaboradores, ${ }^{8}$ os deslocamentos nestas bandas podem ser devido a efeitos de cristalinidade, agregação das partículas e diferença na forma das mesmas que compõe a rede.

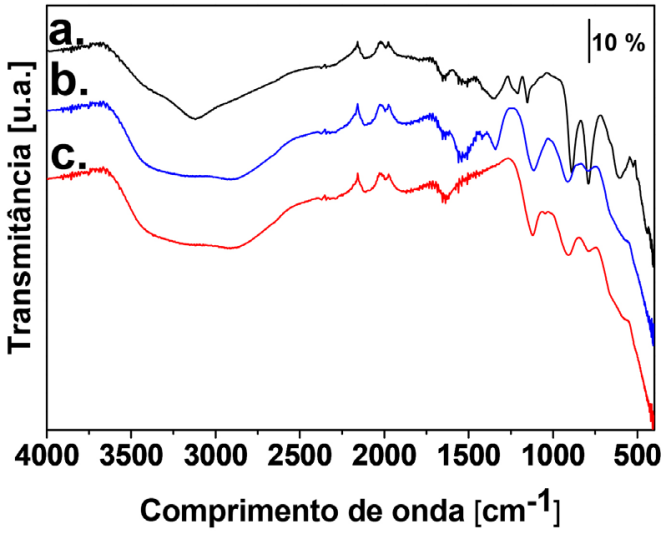

Figura 3. Espectros FTIR para o (a) $\delta$-FeOOH e os seus híbridos (b) $A u(1) /$ $\mathrm{FeOOH} \mathrm{e}(c) \mathrm{Au}(2) / \mathrm{FeOOH}$

Foram realizadas também medidas de espectroscopia de absorção molecular na região do UV-Vis a fim de obter as características ópticas dos materiais. Tanto o $\delta$-FeOOH quanto os híbridos (AuNPs/ $\mathrm{FeOOH}$ ) apresentaram uma banda de absorção alargada na região de 350-750 nm, associada às transições eletrônicas de campo ligante dos íons $\mathrm{Fe}$ (III) no $\mathrm{FeOOH}$ devido ao acoplamento de cátions $\mathrm{Fe}(\mathrm{III})$ adjacentes na estrutura deste oxihidróxido (Figura 4a). ${ }^{34}$ As bandas de absorção na região de 290 a $400 \mathrm{~nm}$ podem ser atribuídas às transições do tipo ${ }^{6} \mathrm{~A}_{1 \mathrm{~g}} \rightarrow{ }^{4} \mathrm{~T}_{1 \mathrm{~g}}\left({ }^{4} \mathrm{P}\right)$ e ${ }^{6} \mathrm{~A}_{1 \mathrm{~g}} \rightarrow{ }^{4} \mathrm{E}\left({ }^{4} \mathrm{D}\right)$. A forte absorção na região próxima a $530 \mathrm{~nm}$ pode ser associada às transições do campo ligante ${ }^{6} \mathrm{~A}_{1 \mathrm{~g}} \rightarrow{ }^{4} \mathrm{E}_{\mathrm{g}},{ }^{4} \mathrm{~A}_{1 \mathrm{~g}}\left({ }^{4} \mathrm{G}\right)$. Bandas de transições $d-d\left({ }^{6} \mathrm{~A}_{1} \rightarrow{ }^{4} \mathrm{~T}_{1}\right)$ dos íons Fe(III) podem ser vistas na região de 800 - $1000 \mathrm{~nm}$ para o material FeOOH.$^{32}$ Foi obtido também o espectro UV-vis dos híbridos AuNPs/FeOOH usando o suporte (FeOOH) como branco (Figura 4b). Nos espectros dos híbridos pode ser observada uma banda em torno de $560 \mathrm{~nm}$ que foi atribuída à banda de ressonância plasmônica das AuNPs com morfologia esférica. ${ }^{15,16,17,35}$ Portanto, estes resultados corroboram com as imagens MEV dos híbridos, indicando que as AuNPs com morfologia esférica foram depositadas na superfície do suporte inorgânico $\delta$-FeOOH. Deve ser também enfatizado que os espectros da Figura 4b (após a subtração do suporte $\mathrm{FeOOH}$ ) são ligeiramente distintos das curvas da Figura 4a (usando $\mathrm{BaSO}_{4}$ como referência), o que pode ser devido à presença das AuNPs e/ou, também, aos efeitos de cristalinidade e tamanho das partículas nas diferentes amostras. ${ }^{8}$ Além disso, é importante salientar que estes compostos híbridos possuem grande capacidade de absorção de luz na região do visível do espectro eletromagnético, sendo interessantes materiais para aplicações em fotocatálise heterogênea ativada por luz solar natural. ${ }^{36}$

\section{Redução catalítica do 4-nitrofenol pelos híbridos AuNPs/FeOOH}

A atividade das nanopartículas de ouro adsorvidas no $\delta$-FeOOH foi investigada usando a redução do composto 4-NP a 4-AP na presença de $\mathrm{NaBH}_{4}$. A Figura 5a apresenta o espectro UV-vis da solução de 4-NP antes da adição do $\mathrm{NaBH}_{4}$, onde pode ser visto uma banda centrada a $317 \mathrm{~nm}$ associada às transições eletrônicas $\pi \rightarrow \pi^{*}$ do anel aromático do 4-nitrofenol. Após a adição de borohidreto de sódio, esta banda é deslocada para a região do vermelho em $400 \mathrm{~nm}$ devido à formação do complexo nitrofenolato. ${ }^{3,5,8,18}$ Esta banda foi usada para monitorar a redução do 4-NP a 4-AP. Sem a adição do catalisador essa banda permanece inalterada por dias indicando que 

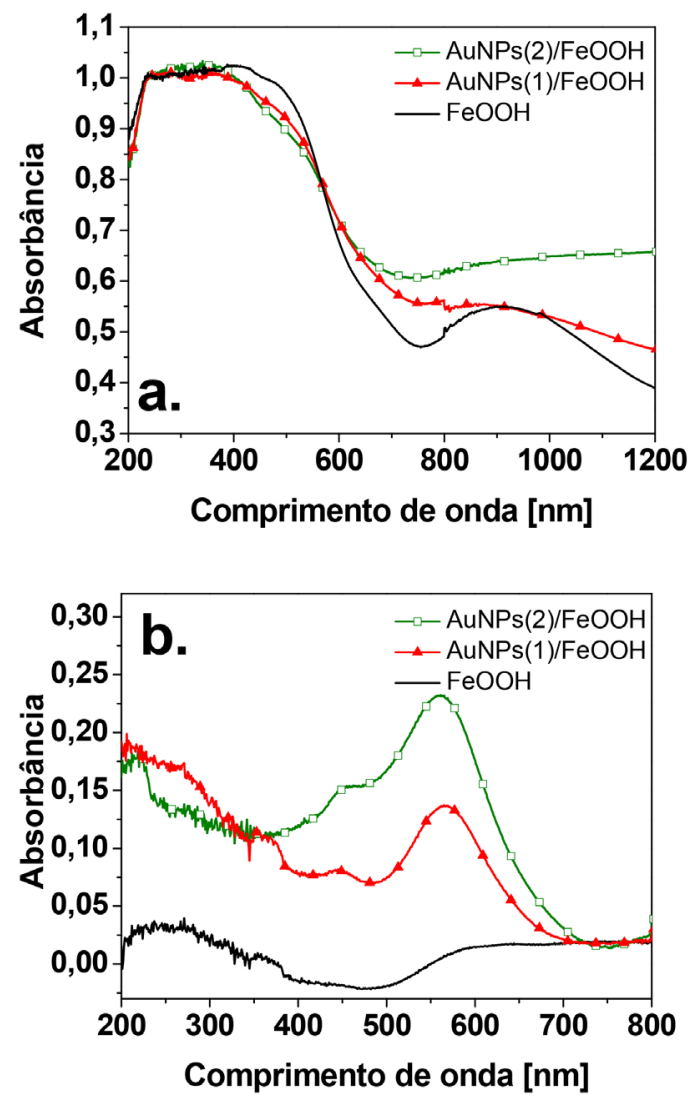

Figura 4. (a) e (b) Espectros de absorção na região do UV-vis-NIR das amostras de $\delta$-FeOOH e de seus híbridos AuNPs/FeOOH. O espectro da Figura $4 b$ foi feito usando o $\mathrm{FeOOH}$ como branco

a redução do composto 4-NP não é cineticamente favorável. Após a adição dos catalisadores AuNPs/FeOOH (Figura 5a-b), rapidamente esta banda é diminuída e, consequentemente, uma nova banda aparece em $298 \mathrm{~nm}$ indicando a formação do 4-aminofenol. ${ }^{3,5,18}$ Isto evidencia que as AuNPs nos híbridos estão atuando como catalisadores para a redução do 4-NP a 4-AP. A redução do 4-NP é completada em torno de 8 e 5 minutos para os catalisadores AuNPs(1)/FeOOH e AuNP(2)/ $\mathrm{FeOOH}$, respectivamente. Estes resultados mostraram que o aumento das AuNPs acelera a redução do composto nitrofenólico, como já observado nos trabalhos de Naik e colaboradores, ${ }^{37}$ que impregnaram NPs de prata nos canais de sílica mesoporosa do tipo SBA-15 para a redução de compostos nitrofenólicos. Nenhuma redução catalítica foi observada na presença apenas do suporte $\delta$-FeOOH puro, indicando que a atividade catalítica pode ser atribuída exclusivamente

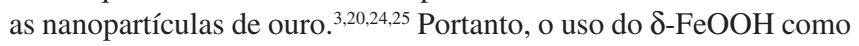
suporte para a deposição de nanopartículas de ouro abre novas perspectivas para o emprego destes materiais como precursores para a produção de híbridos multifuncionais baseados em óxidos de ferro (III) decorados com nanopartículas metálicas através do tratamento térmico dos catalisadores AuNPs/FeOOH..$^{17,26}$

A cinética da reação pode ser facilmente obtida através dos espectros de absorção UV-vis dependentes do tempo (Figura 6). Como a concentração de $\mathrm{NaBH}_{4}$ é muito alta comparada ao 4-NP, pode ser assumido que a reação segue uma cinética de pseudo-primeira ordem. ${ }^{3,5,18}$ A constante de reação de pseudo-primeira ordem $(k)$ para esta redução pode ser fornecida pela equação abaixo:

$$
\left.\ln \left(\mathrm{A}_{\mathrm{t}} / \mathrm{A}_{0}\right)=-k \mathrm{t} \quad \text { (equação } 1\right)
$$

em que $A_{t}$ e $A_{0}$ são os valores das absorbâncias do 4-NP nos
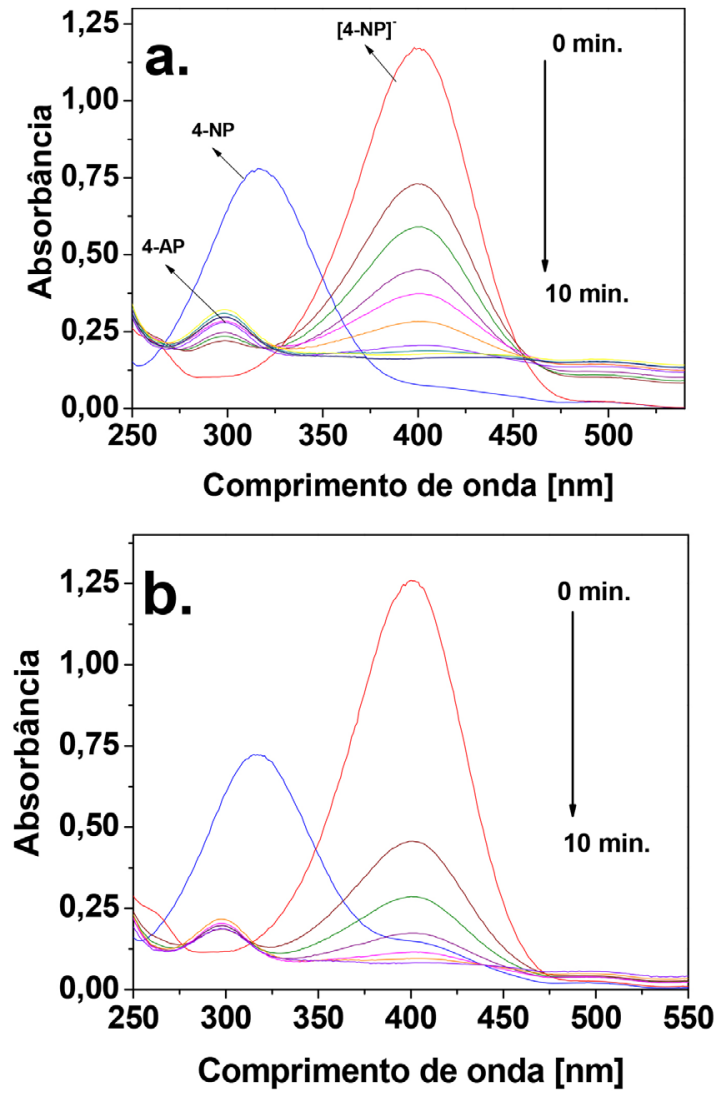

Figura 5 Espectro de absorção UV-vis do 4-NP (curva azul) seguido da adição de $\mathrm{NaBH}_{4}$ e consequente formação do íon 4-nitrofenolato (curva vermelha). As demais medidas foram feitas em intervalos de 1 minuto para acompanhar a redução do 4-NP utilizando os catalisadores (a) AuNPs(1)/ $\mathrm{FeOOH}$ e (b) AuNPs(2)/FeOOH. Na Figura, 4-NP refere-se à banda do 4-nitrofenol, 4-AP refere-se à banda do 4-aminofenol e [4-NP] refere-se à banda do ín nitrofenolato

diferentes intervalos de tempos $(\mathrm{t})$ e no início da reação $\left(\mathrm{t}_{0}\right)$, respectivamente. $\mathrm{O}$ gráfico de $\ln \left(\mathrm{A}_{\mathrm{t}} / \mathrm{A}_{0}\right)$ versus tempo foi linear na presença dos catalisadores AuNPs/FeOOH (Figura 6), obtendo-se uma constante de velocidade $(k)$ de $3,81 \times 10^{-3} \mathrm{~s}^{-1}\left(\mathrm{R}^{2}=0,9979\right)$ e $6,79 \times 10^{-3} \mathrm{~s}^{-1}\left(\mathrm{R}^{2}=0,9806\right)$ para os híbridos AuNPs(1)/FeOOH e AuNPs(2)/FeOOH, respectivamente. Estes resultados estão de acordo com o trabalho recentemente realizado por Walker e Zaleski, ${ }^{38}$ que encontraram uma constante de velocidade de $4,41 \times 10^{-3} \mathrm{~s}^{-1}$ para a magnetita $\left(\mathrm{Fe}_{3} \mathrm{O}_{4}\right)$ decorada com AuNPs. Também foi calculada a frequência de turnover (TOF) para a reação de redução do 4-NP utilizando os híbridos AuNPs/FeOOH como catalisadores. Os valores de TOF foram facilmente obtidos através da equação ${ }^{3} 2$.

$$
\mathrm{TOF}=\frac{\frac{\left[\mathrm{C}_{0}-\mathrm{C}_{\mathrm{t}}\right]}{[\mathrm{Au}]}}{\mathrm{t}}
$$

(equação 2)

Em que $\left[\mathrm{C}_{0}\right]$ corresponde à concentração inicial de 4-nitrofenol em mols, $\left[\mathrm{C}_{\mathrm{t}}\right]$ é referente à concentração de 4-nitrofenol no tempo $t$ ( $\mathrm{t}=5$ min de reação) e $[\mathrm{Au}]$ é a quantidade de AuNPs em mols no catalisador. TOF's de 44,9 e 29,9 $\mathrm{h}^{-1}$ foram obtidos para os catalisadores $\mathrm{AuNPs}(1) / \mathrm{FeOOH}$ e AuNPs(2)/FeOOH, respectivamente. Estes valores de TOF indicaram que os catalisadores apresentam alta atividade catalítica para a redução do 4-NP a 4-AP devido à presença das AuNPs na superfície do FeOOH. ${ }^{3,5,37}$

Os resultados obtidos neste estudo estão de acordo com os estudos 
experimentais usando catalisadores híbridos (por exemplo: NPs de níquel suportadas em carbono ${ }^{39}$ e NPs de ouro adsorvidas em dendrímeros $)^{40}$ para a redução catalítica de 4-NP apresentados na literatura.

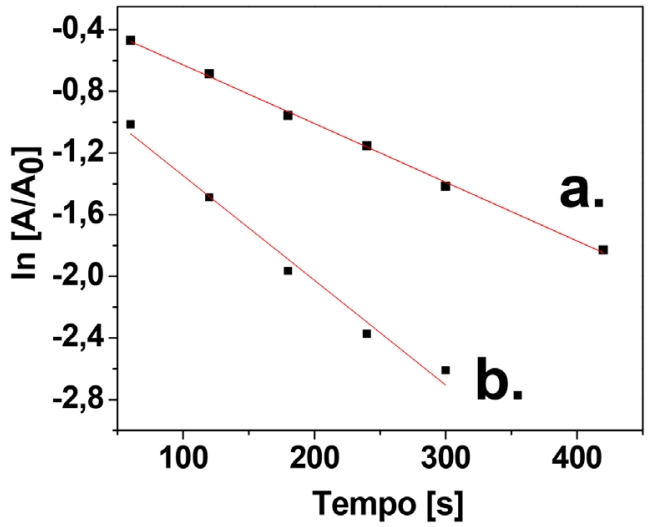

Figura 6. Gráficos da cinética de pseudo-primeira ordem para a reação de redução do 4-nitrofenol na presença dos catalisadores (a) $\mathrm{AuNP}(1) / \mathrm{FeOOH}$ e (b) $\mathrm{AuNP} s(2) / \mathrm{FeOOH}$

\section{CONCLUSÕES}

Materiais híbridos baseados em nanopartículas de ouro e oxihidróxido de ferro (III) foram preparados e caracterizados neste estudo. As nanopartículas de ouro foram depositadas na superfície do $\delta$-FeOOH como observado pelos dados de difração de raios X. Medidas de absorção molecular UV-vis e imagens de microscopia eletrônica também corroboraram com os dados de DRX, indicando que as NPs de ouro possuem morfologia esférica e que elas estão adsorvidas na superfície do suporte inorgânico. Os híbridos AuNPs/ FeOOH foram também empregados como catalisadores na hidrogenação catalítica do poluente orgânico ambiental 4-NP a 4-AP na presença de $\mathrm{NaBH}_{4}$ à temperatura ambiente. A atividade catalítica nos híbridos é aumentada com o aumento da quantidade de NPs de ouro nos catalisadores devido ao aumento da velocidade de reação. Nas condições reacionais estudadas, a redução total do 4-NP ocorreu em torno de 4 min e 8 min para os catalisadores $\operatorname{AuNPs}(2) / \mathrm{FeOOH}$ e $\mathrm{AuNP}(1) / \mathrm{FeOOH}$, respectivamente. Além disso, os materiais híbridos produzidos contendo as fases de $\mathrm{FeOOH}$ e nanopartículas metálicas podem ser interessantes precursores para a preparação de novas nanoestruturas de óxido de ferro decorados com NPs metálicas. Estes compostos podem também ser interessantes materiais para o uso em fotocatálise heterogênea ativada por luz solar devida à alta capacidade de absorção de luz da fase $\delta$-FeOOH na região do visível do espectro eletromagnético.

\section{MATERIAL SUPLEMENTAR}

Figura 1S do Material Suplementar apresenta o espectro EDS-MEV mostrando a distribuição de átomos na superfície do híbrido $\operatorname{AuNPs}(2) / \mathrm{FeOOH}$ : (a) área selecionada e mapas de distribuição do (b) ferro (Fe-K), (d) ouro (Au-K) e (d) oxigênio (O-K); e (e) o espectro EDS-MEV.

\section{AGRADECIMENTOS}

CKSA e EAR agradecem ao $\mathrm{CNPq}$ e à Capes pelas bolsas concedidas. Os autores também agradecem à Fundação de Amparo à Pesquisa ao Estado de Mato Grosso (FAPEMAT, Processo $N^{\circ}$. 222535/2015), ao Estado de Minas Gerais (FAPEMIG), e ao CNPq pelo apoio financeiro que fomentou essa pesquisa.

\section{REFERÊNCIAS}

1. Chang, Y. C.; Chen, D. H.; J. Hazard. Mater. 2009, 165, 664.

2. Zheng, Y.; Shu, J.; Wang, Z.; Mater. Lett. 2015, 158, 339.

3. Quites, F. J.; Azevedo, C. K. S.; Alves, E. P. P.; Germino, J. C.; Vinhas, R. C. G.; Landers, R.; Terezo, A. J.; Atvars, T. D. Z.; J. Braz. Chem. Soc. 2017, 28, 106.

4. Borah, B. J.; Bharali, P.; J. Mol. Catal. A: Chem. 2014, 390, 29.

5. Zhao, P.; Fenga, X.; Huanga, D.; Yanga, G.; Astruc, D.; Coord. Chem. Rev. 2015, 287, 114.

6. Camargo, P. H.; Satyanarayana, K. G.; Wypych, F.; Mater. Res. 2009, $12,1$.

7. Chen, Y.; Zeng, D.; Zhang, K.; Lu, A.; Wang, L.; Peng, D. L.; Nanoscale 2014, 6 .

8. Pedrosa. J.; Costa, B. F.; Portugal, A.; Durães, L.; Mater. Chem. Phys. 2015, 163, 88 .

9. Corrêa, S.; Lacerda, L. C. T.; Pires, M. S.; Rocha, M. V. J.; Nogueira, F. G. E.; da Silva, A. C.; Pereira, M. C.; de Brito, A. D. B.; da Cunha, E. F. F.; Ramalho, T. C.; J. Nanomaterials 2016, 2462135.

10. Lima, L. V. C.; Rodriguez, M.; Freitas, V. A. A.; Souza, T. E.; Machado, A. E. H.; Patrocínio, A. O. T.; Fabris, J. D., Oliveira, L. C. A.; Pereira, M. C.; Appl. Catal., B 2015, 165, 579.

11. Chen, P.; Xu, K.; Li, X.; Guo, Y.; Zhou, D.; Zhao, J.; Wu, X.; Wu, C.; Xie, Y.; Chem. Sci. 2014, 5, 2251.

12. Pereira, M. C.; Garcia, E. M.; Silva, A. C.; Lorençon, E.; Ardisson, J. D.; Murad, E.; Fabris, J. D.; Matencio, T.; Ramalho, T. C.; Rocha, M. V. J.; J. Mater. Chem. 2011, 21, 10280.

13. Silva, A. C.; Almeida, M. R.; Rodriguez, M.; Machado, A. R. T.; Oliveira, L. C. A.; Pereira, M. C.; J. Photochem. Photobiol. A: Chem. 2017, 332, 54

14. Silva, M. F.; Pineda, E. A. G.; Bergamasco, R.; Quim. Nova 2015, 38, 393.

15. Liang, Y. N.; Li, Y.; Ang, C.; Shen, Y.; Chi, D.; Hu, X.; ACS Appl. Mater. Interfaces 2014, 6, 12406.

16. Massola, B. C. P.; Souza, N. M. P.; Stachack, F. F. F.; Oliveira, E. W. R. S.; Germino, J. C.; Terezo, A. J.; Quites, F. J.; Mater. Chem. Phys. 2015, 167, 152.

17. Yue, J.; Jiang, X.; Kaneti, Y. V.; Yu, A.; J. Colloid Interface Sci. 2012, 367, 204.

18. Tang, M.; Huang, G.; Li, X.; Pang, X.; Qiu, H.; Mater. Chem. Phys. 2015, 162, 31 .

19. Silva, A. G. M.; Rodrigues, T. S.; Macedo, A.; Silva, R. T. P.; Camargo, P H. C.; Quim. Nova 2014, 37, 1716.

20. Damasceno, J. P. V.; Maroneze, C. M.; Strauss, M.; Sigoli, F. A.; Mazali, I. O.; New. J. Chem. 2016, 40, 6636.

21. Rossi, L. M.; Costa, N. J. S.; Silva, F. P.; Gonçalves, R. V.; Nanotechnology Rev. 2013, 2, 597.

22. Rossi, L. M.; Costa, N. J. S.; Silva, F. P.; Wojcieszak, R.; Green Chem. 2014, 16, 2906.

23. Woo, H.; Park, K. H.; Catal. Commun. 2014, 46, 133.

24. Wang, Z. Z.; Zhai1, S. R.; Zhai, B.; An, Q. D.; Li, S. W.; J. Sol-Gel Sci. Technol. 2015, 75, 680.

25. Walker, J. M.; Zaleski, J. M.; Nanoscale 2016, 8, 1535.

26. Guoa, X.; Liua, Q.; Wanga, L.; Huanga, H.; Yanga, D.; Cheng, M. L.; Mater. Sci. Eng. B 2012, 177, 321.

27. Bian, Z.; Tachikawa, T.; Zhang, P.; Fujitsuka, M.; Majima, T.; J. Am. Chem. Soc. 2014, 136, 458.

28. Wu, S.; Lu, J.; Ding, Z.; Li, N.; Fu, F.; Tang, B.; RSC Adv. 2016, 6, 82118.

29. Wang, Y-H.; Chen, K-C.; Int. J. Environ. Res. Public Health 2014, 11, 9325.

30. Marinho, J. Z.; Montes, R. H. O.; de Moura, A. P.; Longo, E.; Varela, J. A.; Munoz, R. A. A.; Lima, R. C.; Mater. Res. Bull. 2014, 49, 572. 
31. Maiti, D.; Manju, U.; Velaga, S.; Devi, P. S.; Cryst. Growth Des. 2013 , 13, 3637.

32. Villalba, J. C.; Constantino, V. R. L.; Anaissi, F. J.; J. Colloid Interface Sci. 2010, 349, 49.

33. Carlson, L.; Schwertmann, U.; Clay Minerals Soc. 1980, $28,272$.

34. Martin, T. P.; Merlin, R.; Huffman, D. R.; Cardona, M.; Solid State Commun. 1977, 22, 565.

35. Hamdy, M. S.; Micropor. Mesopor. Mater. 2016, $220,81$.
36. Oliveira, L. C. A.; Fabris, J. D.; Pereira, M. C.; Quim. Nova 2013, 36, 123.

37. Naik, B.; Hazra, S.; Prasad, V. S.; Ghosh, N. N.; Catal. Commun. 2011, 12,1104

38. Walker, J. M.; Zaleski, J. M.; Nanoscale, 2016, 8, 1535.

39. Xia, J.; He, G.; Zhang, L.; Sun, X.; Wang, X.; Appl. Catal. B: Environ. 2016, 180,408

40. Nemanashi, M.; Meijboom, R.; J. Colloid Interface Sci. 2013, 389, 260. 